\title{
Severe allergic reactions after COVID-19 vaccination with the Pfizer/BioNTech vaccine in Great Britain and USA
}

\author{
Position statement of the German Allergy Societies: Medical Association of \\ German Allergologists (AeDA), German Society for Allergology and Clinical \\ Immunology (DGAKI) and Society for Pediatric Allergology and Environmental \\ Medicine (GPA)
}

\begin{abstract}
Ludger Klimek · Natalija Novak · Eckard Hamelmann · Thomas Werfel · Martin Wagenmann · Christian Taube · Andrea Bauer · Hans Merk · Uta Rabe · Kirsten Jung · Wolfgang Schlenter · Johannes Ring · Adam Chaker · Wolfgang Wehrmann · Sven Becker · Norbert Mülleneisen · Katja Nemat · Wolfgang Czech · Holger Wrede • Randolf Brehler · Thomas Fuchs · Thilo Jakob · Tobias Ankermann · Sebastian M. Schmidt · Michael Gerstlauer • Christian Vogelberg · Thomas Zuberbier · Karin Hartmann · Margitta Worm
\end{abstract}

Summary Two employees of the National Health Service (NHS) in England developed severe allergic reactions following administration of BNT162b2 vaccine against COVID-19 (coronavirus disease 2019). The British SmPC for the BNT162b2 vaccine already includes reference to a contraindication for use in individuals who have had an allergic reaction to the vaccine or any of its components. As a precautionary

\section{Klimek}

Center for Rhinology and Allergology Wiesbaden,

Wiesbaden, Germany

\section{N. Novak}

Clinic and Polyclinic for Dermatology and Allergology,

University Hospital Bonn, Bonn, Germany

\section{E. Hamelmann}

Pediatric and Adolescent Medicine, Children's Center Bethel, University Hospital OWL of Bielefeld University, Bielefeld, Germany

\section{T. Werfel}

Department of Dermatology, Allergology and Venereology, Hannover Medical School, Hannover, Germany

\section{Wagenmann}

Department of Ear, Nose and Throat Medicine, University

Hospital Düsseldorf, Düsseldorf, Germany

C. Taube

Clinic for Pneumology, University Medicine

Essen-Ruhrlandklinik, West German Lung Center, Essen, Germany measure, the Medicines and Healthcare products Regulatory Agency (MHRA) has issued interim guidance to the NHS not to vaccinate in principle in "patients with severe allergies". Allergic reactions to vaccines are very rare, but vaccine components are known to cause allergic reactions. BNT162b2 is a vaccine based on an mRNA embedded in lipid nanoparticles and blended with other substances to enable its transport

\footnotetext{
A. Bauer

Clinic and Polyclinic for Dermatology, University Allergy Center, University Clinic Carl Gustav Carus, Technical University Dresden, Dresden, Germany

H. Merk

Department of Dermatology and Allergology, RWTH Aachen, Aachen, Germany

U. Rabe

Clinic for Allergology, Johanniter Hospital in Fläming Treuenbrietzen GmbH, Treuenbrietzen, Germany

K. Jung

Practice for Dermatology, Immunology and Allergology, Erfurt, Germany

W. Schlenter

Medical Association of German Allergologists, Dreieich, Germany

J. Ring

Skin and Laser Center at the Opera, Munich, Germany
} 
into the cells. In the pivotal phase III clinical trial, the BNT162b2 vaccine was generally well tolerated, but this large clinical trial, used to support vaccine approval by the MHRA and US Food and Drug Administration, excluded individuals with a "history of a severe adverse reaction related to the vaccine and/or a severe allergic reaction (e.g., anaphylaxis) to a component of the study medication". Vaccines are recognized as one of the most effective public health interventions. This repeated administration of a foreign protein (antigen) necessitates a careful allergological history before each application and diagnostic clarification and a risk-benefit assessment before each injection. Severe allergic reactions to vaccines are rare but can be life-threatening, and it is prudent to raise awareness of this hazard among vaccination teams and to take adequate precautions while more experience is gained with this new vaccine.

Keywords BNT162b2 - Severe allergic reactions · Anaphylaxis $\cdot$ Vaccines $\cdot$ mRNA

$\begin{array}{ll}\text { Abbreviations } \\ \text { EUA } & \text { Emergency Use Authorization } \\ \text { FDA } & \text { Food and Drug Administration }\end{array}$

\section{A. Chaker}

Ear, Nose and Throat Clinic, Klinikum rechts der Isar, Technical University of Munich, Munich, Germany Center for Allergy and Environment (ZAUM), Klinikum rechts der Isar, Technical University of Munich, Munich, Germany

W. Wehrmann

Dermatological Group Practice Wehrmann, Münster, Germany

\section{S. Becker}

Ear, Nose and Throat Clinic, University of Tübingen, Tübingen, Germany

N. Mülleneisen

Asthma and Allergy Center Leverkusen, Leverkusen, Germany

\section{K. Nemat}

Practice for Pediatric Pneumology/Allergology at the Children's Center Dresden (Kid), Dresden, Germany

\section{W. Czech}

Practice and Clinic for Allergology/Dermatology Schwarzwald-Baar Clinic, Villingen-Schwenningen, Germany

\section{H. Wrede}

Ear, Nose and Throat Specialist, Herford, Germany

\section{R. Brehler}

Department of Skin Diseases, Outpatient Clinic for Allergology, Occupational Dermatology and Environmental Medicine, Münster University Hospital, Münster, Germany

\section{T. Fuchs}

Department of Dermatology, Venereology and Allergology, University Medical Center, Georg-August University, Göttingen, Germany
LNP
Lipid-based nano particles
MHRA
Medicines and Healthcare products Reg- ulatory Agency
NHS National Health Service
PEG Polyethylenglycol
SmPC Summary of Product Characteristics
S-Protein Spike-protein

\section{Background}

On December 9, 2020, the Medicines and Healthcare products Regulatory Agency (MHRA) in the United Kingdom informed of severe allergic reactions in two employees of the National Health Service (NHS) in England following administration of BNT162b2 vaccine against COVID-19 (Coronavirus disease 2019). Both patients had a history of anaphylaxis, and as far as is currently known both recovered rapidly and completely from these severe allergic reactions. Since it is not clear which component of the vaccine triggered the reaction, an investigation was initiated to fully understand the two incidents and their causes.

The British summary of product characteristics (SmPC) for the BNT162b2 vaccine already includes reference to a contraindication for use in individuals who have had an allergic reaction to the vaccine

\section{T. Jakob}

Department of Dermatology and Allergology, University Hospital Gießen, UKGM Justus Liebig University Gießen, Gießen, Germany

\section{T. Ankermann}

Clinic for Pediatric and Adolescent Medicine, Pneumology, Allergology, Neonatology, Intensive Care Medicine, Infectiology, Schleswig-Holstein University Medical Center, Kiel, Germany

\section{S. M. Schmidt}

Center for Pediatric and Adolescent Medicine, Clinic and Polyclinic for Pediatric and Adolescent Medicine, University Medicine Greifswald, Greifswald, Germany

\section{Gerstlauer}

Pediatric Pneumology/Pediatric Allergology, 2nd Clinic for Children and Adolescents, University Hospital Augsburg, Augsburg, Germany

\section{Vogelberg}

Department of Pediatric Pneumology/Allergology, Clinic and Polyclinic for Pediatric and Adolescent Medicine, Carl Gustav Carus University Hospital, Dresden, Germany

\section{T. Zuberbier}

Clinic for Dermatology, Venereology and Allergology, Charité-University Medicine Berlin, Berlin, Germany

\section{K. Hartmann}

Clinic for Dermatology and Allergology, University Hospital Basel, Basel, Switzerland

Univ.-Prof. Dr. M. Worm ( $₫)$

Allergologie und Immunologie, Klinik für Dermatologie, Venerologie und Allergologie, Charité - Universitätsmedizin Berlin, Campus Mitte, Charitéplatz 1, 10117 Berlin, Germany margitta.worm@charite.de 
or any of its components [1]. As a precautionary measure, the MHRA has issued interim guidance to the NHS not to vaccinate in principle "patients with severe allergies". It is likely that the restriction on indications now imposed by the MHRA will result in significantly fewer patients being able to receive the vaccine.

Further adverse reaction reports can be expected from the U.S. Food and Drug Administration's (FDA) Emergency Use Authorization (EUA) for BNT162b2 granted on December 11, 2020, as very large vaccination numbers are now likely to be achieved rapidly.

\section{Allergic reactions to vaccines}

Allergic reactions to vaccines are very rare, occurring at 1 per $1,000,000$ to 30 per 100,000 vaccinations [2-6].

Vaccine components known to cause allergic reactions include residues of animal proteins, antimicrobial agents, preservatives, stabilizers, and adjuvants in addition to the active component of the vaccine (the actual antigen) that elicit the immune response [2-6]. Individual vaccine components associated with causing vaccine anaphylaxis include chicken egg protein, gelatin, cow's milk proteins, and other additives and trace compounds left over from the manufacturing process, in addition to latex components from the sealing plugs in multiple vaccine vials [3-6].

\section{The vaccine BNT162b2}

BNT162b2 is "temporarily licensed" in the United Kingdom for active immunization to prevent COVID19 disease caused by the SARS-CoV-2 virus in persons 16 years of age and older [7].

The vaccine is administered intramuscularly in two doses of $0.3 \mathrm{ml}$ each, 21 days apart. Thawed COVID-19 mRNA vaccine BNT162b2 requires dilution in its original vial $(0.45 \mathrm{ml})$ with $1.8 \mathrm{ml}$ of unpreserved sodium chloride $0.9 \%$ solution for injection, prior to withdrawing a $0.3 \mathrm{ml}$ dose $[7,8]$.

The listed excipients in BNT162b2 are ALC-0315 ((4-hydroxybutyl)azanediyl)bis (hexane-6,1-diyl)bis(2hexyl decanoate), ALC-0159 (2-((polyethylene glycol)2000)-N,N-ditetradecylacetamide), 1,2-distearoyl-snglycero-3-phosphocholine, cholesterol, potassium chloride, potassium dihydrogen phosphate, sodium chloride, disodium hydrogen phosphate dihydrate, sucrose, and water for injection $[7,8]$.

One vial $(0.45 \mathrm{ml})$ contains five doses of $30 \mu \mathrm{g}$ of highly purified, single-stranded, 5'-capped mRNA (BNT162b2 RNA) produced by cell-free in vitro transcription on an appropriate DNA template and encoding the viral spike(S) protein of SARS-CoV-2 [7, 8].

This mRNA is embedded in lipid nanoparticles. mRNA is rapidly degraded by ribonucleases, readily taken up by mononuclear phagocytes, and has poor ability to penetrate cell membranes due to its negative electrical charge and high molecular weight. Therefore, mRNA requires a protective envelope for use as a vaccine. In the BioNTech vaccine, lipid-based nanoparticles (LNPs) are used as nonviral vectors for this purpose, containing cationic lipids that coat the polyanionic mRNA with its tertiary or quaternary amines, complemented by zwitterionic lipids that mimic cell membrane phospholipids, and cholesterol that stabilizes the lipid bilayer of the nanoparticle. Finally, polyethylene glycol (PEG)-modified lipids enable the assembly of a hydrate shell, increasing the solubility of the LNPs.

PEG or macrogol is a polyether compound commonly used as an additive in cosmetics, pharmaceuticals, and also in foods [9]. PEG exists in molecular weight types ranging from $200 \mathrm{~g} / \mathrm{mol}$ to $10,000,000 \mathrm{~g} / \mathrm{mol}$ and allergic reactions have been reported after its use in a variety of drugs and cosmetic products $[10,11]$. There is cross-reactivity to polysorbate 80 due to common chemical motifs [12, 13]. Allergic reactions to PEG are probably diagnosed too rarely, so PEG is also considered a "hidden" allergen [11]. Severe allergic reactions have been described in diagnostic skin testing, so this should only be performed in allergy-specialized centers according to published standard regimens.

In the pivotal phase III clinical trial, the BNT162b2 vaccine was generally well tolerated [8]. A total of 43,548 participants were randomized, of whom 43,448 received one injection, 21,720 with BNT162b2 and 21,728 with placebo 18,556 received a second dose of BNT162b2 [8]. The most common adverse reactions were local reactions at the injection site $(84.7 \%)$, fatigue $(62.8 \%)$, headache $(55.1 \%)$, muscle pain $(38.3 \%)$, chills $(31.9 \%)$, joint pain $(23.6 \%)$, fever $(14.2 \%)[7,8]$. Most reactions were mild to moderate in severity. Severe adverse reactions occurred in up to $4.6 \%$ and were more common after the second dose and less common in adults $>55$ years of age. Lymphadenopathy was reported in $0.3 \%$. Systemic adverse reactions were usually mild or moderate in severity, generally occurring the day after vaccination and lasting one to two days thereafter $[7,8]$. The occurrence of allergic reaction was reported in similar numbers in both the vaccine-verum and placebo groups $(0.63 \%$ vs. $0.51 \%)[7,8]$. However, this large clinical trial, used to support vaccine approval by the MHRA and FDA, excluded individuals with a "history of a severe adverse reaction related to the vaccine and/or a severe allergic reaction (e.g., anaphylaxis) to a component of the study medication" [7, 8]. Based on this the UK package insert based on this states that the vaccine should not be administered to individuals who are allergic to the active ingredient or any of the other ingredients listed. In this respect, the patient information complies with the exclusion criteria of the clinical trial. 


\section{Evaluation and outlook}

Vaccines are recognized as one of the most effective public health interventions. The primary goal of vaccination programs is the protection of the vaccinated individuals. In addition, in many cases they also aim to protect unvaccinated individuals. The ultimate goal is creating herd immunity, i.e., resistance to the spread of a contagious disease in a population, which occurs when a sufficiently high proportion of individuals are immune to that disease, especially through vaccination [14].

The development of such herd immunity requires vaccination rates of $>60 \%$ of the total population [14].

However, to achieve effective individual immunization of $>95 \%$ with BNT162b2, a second vaccination ("booster") is necessary. This repeated administration of a foreign protein (antigen) necessitates a careful allergological history before each application and diagnostic clarification, if necessary, and risk-benefit assessment before each injection.

Against this background we state that

- Patients and people who are to receive a vaccination against COVID-19 must also be regularly informed about possible severe allergic/anaphylactic reactions and questioned with regard to such incidents in the past.

- Allergic reactions to additives, in particular PEG and cross-reactive PEG analogues, must be systematically queried in order to identify patients at risk.

- In suspected cases, allergological clarification (skin prick test, laboratory diagnostics) and consultation of an allergist should be carried out.

- Personnel performing vaccination against COVID19 must always be prepared for the possibility of severe allergic/anaphylactic reactions and vaccination teams and vaccination centers should be trained in the treatment of anaphylaxis according to the recommendations of the current AWMF guideline on the acute therapy and management of anaphylaxis [15].

- It will be important to understand the specific cause of the two reported severe allergic reactions and the medical history of the individuals involved so that any risks of allergic reactions can be more accurately defined and, if possible, circumvented.

- Current authority guidelines in the UK exclude patients with severe allergies from vaccination with BNT162b2.

- More precise definitions of the type, cause, and severity of severe allergic reactions are needed because, given the high incidence of patients with "severe" allergies (a significant proportion of the total population in Europe and the US, depending on the definition), excluding all such patients from vaccination could have a significant impact on achieving the goal of herd immunity. On the other hand, a more precise definition (e.g., "ana- phylaxis-prone patients") would suggest only $1-3 \%$ of the population for whom vaccination would be impossible or only possible with special protective measures.

- More data need to be collected from both clinical trials and clinical practice that will improve our knowledge of the safety profile of COVID-19 vaccines, particularly with regard to severe allergic reactions.

Severe allergic reactions to vaccines are rare but can be life-threatening, and it is prudent to raise awareness of this hazard among vaccination teams and to take adequate precautions while more experience is gained with this new vaccine. Patients with a history of severe allergic reactions may be vaccinated with adequate medication and by physicians experienced in the management of anaphylactic reactions if the ongoing investigation in the UK allows this recommendation. PEG is currently considered to have most likely triggered the severe allergic reactions in the 2 affected patients in the UK, but further investigations are awaited.

If confirmed, it would only be necessary to exclude patients with known allergic reactions to PEG, PEG analogs, and other additives from vaccination with BNT162b2, but not all patients with a history of severe allergic reactions, which would significantly expand the pool of potentially vaccinatable individuals.

For BNT162b2, safety monitoring will continue for 2 years after administration of the second vaccine dose within the study [8].

Funding Open Access funding enabled and organized by Projekt DEAL

Conflict of interest L. Klimek reports grants and personal fees from Allergopharma, grants and personal fees from MEDA/ Mylan, personal fees from HAL Allergie, personal fees from ALK Abelló, grants and personal fees from LETI Pharma, grants and personal fees from Stallergenes, grants from Quintiles, grants and personal fees from Sanofi, grants from ASIT biotech, grants from Lofarma, personal fees from Allergy Therapeut., grants from AstraZeneca, grants and personal fees from GSK, grants from Inmunotek, personal fees from Cassella med, personal fees from Novartis, outside the submitted work; and Membership: AeDA, DGHNO, Deutsche Akademie für Allergologie und klinische Immunologie, HNOBV, GPA, EAACI. K. Hartmann has received research funding from Euroimmun and Thermofisher and consultancy or lecture fees from Allergopharma, ALK-Abello, Blueprint, Deciphera, Menarini, Novartis and Takeda. J. Ring reports personal fees from Mylan, personal fees from Allergika, outside the submitted work. A. Chaker reports grants and other from Allergopharma, grants and other from ALK Abello, grants and other from Bencard/Allergen Therapeutics, grants and other from ASIT Biotech, other from Lofarma, grants and other from GSK, grants and other from Novartis, grants and other from LETI, grants and other from Roche, grants and other from Zeller, other from Sanofi Genzyme, grants from European Institute of Technology, grants and other from AstraZeneca, grants and other from Immunotek, outside the submitted work; in addition, Dr. Chaker has a patent A ratio of immune 
cells as prognostic indicator of therapeutic success in allergenspecificimmunotherapy: 17177681 .8licensed to none. M.Wagenmann has received fees for advice, lectures or research support from the following companies in the past 3 years: ALK-Abelló, Allergopharma, AstraZeneca, Bencard Allergie, Genzyme, GSK, HAL Allergie, Infectopharm, LETI Pharma, MEDA Pharma, Novartis, Regeneron, Sanofi Aventis, Stallergenes, Teva-all outside of the present work. T. Ankermann has received fees and accommodation and travel expenses for lectures and publications from the following companies and institutions: Abbvie, Aimmune, Allergopharma, Chiesi, Infectopharm, Novartis, UKSH Academy, RG, Springer publishing house, Scientific publishing company, GPP e.V, GPA e.V., nappa e. V., ÖGKJ e.V. Dr. Ankermann has received fees for advisory boards from Boehringer Ingelheim, Aimmune, and fees for safety board from Allergopharma, all outside of the present work. C. Vogelberg has received lecture fees or consultant fees or travel and accommodation costs from: ALK, Allergopharma, Aimmune, DBV, LETI, Novartis, Stallergenes, HAL, Bencard, Sanofi, Aimmune, GPA e.V., APPA e. V. all outside of the present work. T. Werfel reports grants and/or fees from AbbVie, ALK Abello, Almirall, Astellas, Bencard, Galderma, Janssen/JNJ, Leo Pharma, Leti, Lilly, Novartis, Pfizer, Regeneron/Sanofi, Stallergen, all outside of the present work. A. Bauer reports grants and personal fees from Novartis, Leo Pharma, Sanofi/Regeneron, Shire/Takeda, Genentech, outside the submitted work. T. Zuberbier reports personal fees and/or fees for talk from Bayer Health Care, FAES, Novartis, Henkel, AstraZeneca, AbbVie, ALK, Almirall, Astellas, Bencard, Berlin Chemie, HAL, Leti, Meda, Menarini, Merck, MSD, Pfizer, Sanofi, Stallergenes, Takeda, Teva, UCB, Kryolan, L'Oréal outside the submitted work. T. Jakob reports grants, personal fees and non-financial support from Novartis, grants, personal fees and non-financial support from ALK-Abello, personal fees and non-financial support from Allergy Therapeutics/Bencard, personal fees from Allergopharma, personal fees from Thermo Fisher, outside the submitted work. M. Worm reports honorarium for advisory boards and lecture activities from Regeneron Pharmaceuticals, DBV Technologies S.A, Stallergenes GmbH, HAL Allergie GmbH, Bencard Allergie $\mathrm{GmbH}$, Allergopharma GmbH \& Co. KG, ALK-Abelló Arzneimittel GmbH, Mylan Germany GmbH, Leo Pharma $\mathrm{GmbH}$, Sanofi-Aventis Deutschland $\mathrm{GmbH}$, Aimmune Therapeutics UK Limited, Actelion Pharmaceuticals Deutschland $\mathrm{GmbH}$, Novartis AG, Biotest AG, AbbVie Deutschland GmbH \& Co. KG, Lilly Deutschland $\mathrm{GmbH}$, outside the submitted work. N. Novak, E. Hamelmann, C. Taube, H. Merk, U. Rabe, K. Jung, W. Schlenter, W. Wehrmann, S. Becker, N. Mülleneisen, K. Nemat, W. Czech, H. Wrede, R. Brehler, T. Fuchs, S. M. Schmidt and M. Gerstlauer declare that they have no competing interests.

Open Access This article is licensed under a Creative Commons Attribution 4.0 International License, which permits use, sharing, adaptation, distribution and reproduction in any medium or format, as long as you give appropriate credit to the original author(s) and the source, provide a link to the Creative Commons licence, and indicate if changes were made. The images or other third party material in this article are included in the article's Creative Commons licence, unless indicated otherwise in a credit line to the material. If material is not included in the article's Creative Commons licence and your intended use is not permitted by statutory regulation or exceeds the permitted use, you will need to obtain permission directly from the copyright holder. To view a copy of this licence, visit http://creativecommons.org/licenses/by/4.0/.

\section{References}

1. England NHS. Patient Group Direction for COVID-19 mRNA vaccine BNT162b2 (Pfizer/BioNTech)-National COVID-19 Vaccination Programme. England NHS. 2020. www.england.nhs.uk/coronavirus/wp-content/uploads/ sites/52/2020/12/C0956-Patient-Group-Direction-PfizerBioNTech-COVID-Vaccine-Summary-document-11-De cember-2020.pdf. Accessed 15 Dec 2020.

2. Caubet J-C, Ponvert C. Vaccine allergy. Immunol Allergy Clin North Am. 2014;34:597-613.

3. Dreskin SC, Halsey NA, Kelso JM, Wood RA, Hummell DS, Edwards KM, et al. International Consensus (ICON): allergic reactions to vaccines. World Allergy Organ J. 2016;9:32.

4. McNeil MM, DeStefano F. Vaccine-associated hypersensitivity. JAllergy Clin Immunol. 2018;141:463-72.

5. Nilsson L,BrockowK,AlmJ,CardonaV,CaubetJ-C,GomesE, et al. Vaccination and allergy: EAACI position paper, practical aspects. Pediatr Allergy Immunol. 2017;28:628-40.

6 . Wood RA. Allergic reactions to vaccines. Pediatr Allergy Immunol. 2013;24:521-6.

7. England, NHS. England, NHS.. www.england.nhs.uk. Accessed 15Dec 2020.

8. Polack FP, Thomas SJ, Kitchin N, Absalon J, Gurtman A, Lockhart S, et al. Safety and efficacy of the BNT162b2 mRNA Covid-19 vaccine. N Engl J Med. 2020; https://doi.org/10. 1056/NEJMoa2034577.

9. Pizzimenti S, Heffler E, Gentilcore E, Raie A, Bussolino C, Nebiolo F, et al. Macrogol hypersensitivity reactions during cleansing preparation for colon endoscopy. J Allergy Clin Immunol Pract. 2014;2:353-4.

10. Wenande E, Garvey LH. Immediate-type hypersensitivity to polyethylene glycols: a review. Clin Exp Allergy. 2016;46:907-22.

11. Wylon K, Dölle S, Worm M. Polyethylene glycol as a cause of anaphylaxis. Allergy Asthma Clin Immunol. 2016;12:67.

12. Stone CA, Liu Y, Relling MV, Krantz MS, Pratt AL, Abreo A, et al. Immediate hypersensitivity to polyethylene glycols and polysorbates: more common than we have recognized. JAllergy Clin Immunol Pract. 2018;7:1533-1540.e8.

13. Zhou Z-H, Stone CA, Jakubovic B, Phillips EJ, Sussman G, Park J, et al. Anti-PEG IgE in anaphylaxis associated with polyethylene glycol. J Allergy Clin Immunol Pract. 2020; https://doi.org/10.1016/j.jaip.2020.11.011.

14. Scarbrough Lefebvre CD, Terlinden A, Standaert B. Dissecting the indirect effects caused by vaccines into the basic elements. Hum Vaccin Immunother. 2015;11:2142-57.

15. Ring J, Beyer K, Biedermann T, Bircher A, Fischer M, Fuchs $\mathrm{T}$, et al. Guideline (S2k) on acute therapy and management of anaphylaxis: 2021 update. S2k-Guideline of the German Society for Allergology and Clinical Immunology (DGAKI), the Medical Association of German Allergologists (AeDA), the Society of Pediatric Allergology and Environmental Medicine (GPA), the German Academy of Allergology and Environmental Medicine (DAAU), the German Professional Association of Pediatricians (BVKJ), the Society for Neonatology and Pediatric Intensive Care (GNPI), the German Society of Dermatology (DDG), the Austrian Society for Allergology and Immunology (ÖGAI), the Swiss Society for Allergy and Immunology (SGAI), the German Society of Anaesthesiology and Intensive Care Medicine (DGAI), the German Society of Pharmacology (DGP), the German Respiratory Society (DGP), the patient organization German Allergy and Asthma Association (DAAB), the German Working Group of Anaphylaxis Training and Education (AGATE) Allergo J Int 2021;30:1-25. 